\title{
Giant Optical Activity of Helical Architectures of Plasmonic Nanorods
}

\author{
Aristi Christofi, ${ }^{*}{ }^{\dagger}+$ Nikolaos Stefanou, $^{\dagger}$ Georgios Gantzounis, ${ }^{\|}$and Nikolaos Papanikolaou ${ }^{\S}$ \\ ${ }^{\dagger}$ University of Athens, Section of Solid State Physics, Panepistimioupolis, GR-157 84 Athens, Greece \\ ${ }^{\ddagger}$ Department of Theoretical Physics of Multielectron and Magnetic Systems and ${ }^{\S}$ Department of Microelectronics, Institute of \\ Advanced Materials, Physicochemical Processes, Nanotechnology and Microsystems of Materials Science, NCSR "Demokritos", \\ GR-153 10 Athens, Greece \\ "Graduate Aeronautical Laboratories (GALCIT) and Department of Applied Physics, California Institute of Technology, Pasadena, \\ California 91125, United States
}

ABSTRACT: A systematic study, by means of full electrodynamic calculations, of the optical activity of layer-by-layer chiral crystals of finite silver nanorods is presented. The nature of the eigenmodes of the electromagnetic field and the formation of partial gaps for a specific circular polarization in these crystals are analyzed by reference to the hybrid plasmon modes of the structural basis of twisted nanorods. It is shown that collective plasmon modes of the helical assembly give rise to giant optical activity effects, which persist for any angle of incidence and polarization direction. The effects, which are robust against the twisting angle and become more pronounced with increasing particle concentration, can be tuned within a broad range of frequencies in the infrared and visible spectrum by appropriately choosing the rod length. Potential applications of these structures for polarization control in subwavelength optical components are anticipated.

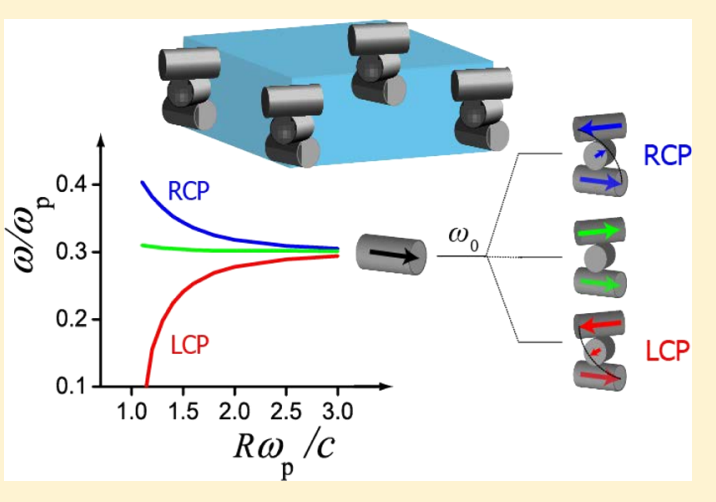

\section{INTRODUCTION}

Collective chirality and the associated optical activity in nanostructures, including chiral assemblies of nonchiral particles, either randomly distributed in solution or periodically arranged on a crystal lattice, attract increasing interest in recent years. Natural chiral molecules such as proteins and DNA show considerable optical activity in the ultraviolet and infrared ranges owing to the electronic and vibrational excitations of their chiral secondary structure. ${ }^{1}$ Cholesteric liquid crystals exhibit strong circular dichroism and singular dispersion of the rotatory power due to a circular Bragg phenomenon, that is, a strong reflectance only for circularly polarized light of the same handedness as that of the physical structure of the chiral medium. ${ }^{2}$ This effect suggests potential for use of these crystals in numerous applications, such as low-threshold mirrorless lasing, circularly polarized emission, ${ }^{3}$ and compact thin-film optical diodes. ${ }^{4}$ Optical activity of artificial helical nanostructures has been addressed in the context of photonic crystals, ${ }^{5}$ and advances in fabrication techniques allowed for the realization of a diversity of thin-film and bulk chiral structures that exhibit enhanced optical activity and sizable partial photonic band gaps for just one of the two circular polarizations. $^{6-14}$

Artificial plasmonic architectures consisting of either chiral metallic building units ${ }^{15-22}$ or nonchiral metallic nanoparticles arranged in chiral geometries such as pyramids, tetrahedra, helices, and so on ${ }^{23-27}$ provide unique opportunities to achieve extraordinary optical activity effects, which can be tuned within a wide range from near-infrared to ultraviolet frequencies and offer impressive possibilities, among others, in the design of subwavelength components for polarization-control applications in miniaturized optical devices. In the case of nanoparticle complexes, these strong effects originate most often from collective plasmon-plasmon interactions of the nanoparticles, whereas assembling such chiral plasmonic metamolecules into periodic structures ${ }^{28-30}$ enriches the possibilities for tailoring their optical response. In particular, it has been reported that a robust particle-plasmon-mediated circular dichroism is obtained for a chiral complex bearing elongated particles, which are oriented along a helix. ${ }^{26}$ Research on chiral plasmonic architectures is at the frontier of nanophotonics also because of potential applications in biology, ${ }^{31}$ chemistry, ${ }^{32,33}$ and optics of novel metamaterials. ${ }^{34}$ These structures can be realized in the laboratory using modern nanofabrication methods like lithography, ${ }^{15-22,28-30}$ molecular self-assembly, ${ }^{35,36}$ and DNAand peptide-directed assembly, ${ }^{37-39}$ whereas, recently, the potential of the DNA origami approach for the programmable and nanometer-precise design of helical assemblies of metallic nanoparticles was also demonstrated. ${ }^{40,41}$

In previous work on a specific design of a spiral-staircase layer-by-layer structure of metallic nanorods studying negative refraction, we revealed the occurrence of strong optical activity for light of specific polarization incident normal to the layers. ${ }^{42}$ In the present article, we go further in this direction and report

Received: $\quad$ May 21, 2012

Revised: July 3, 2012

Published: July 10, 2012 
a thorough investigation of the optical activity in this class of $3 \mathrm{D}$ helical periodic structures by means of rigorous full electrodynamic calculations using the layer-multiple-scattering (LMS) method, ${ }^{43-45}$ properly extended to describe axissymmetric particles with arbitrary orientation. Photonic band diagrams along an arbitrary direction, in conjunction with relevant polarization-resolved transmission spectra, are analyzed by reference to the hybrid plasmonic orbitals ${ }^{47}$ of the chiral nanorod complex that constitutes the basis of the crystal structure. Subsequently, circular dichroism and birefringence effects within the visible spectrum are systematically studied for a series of such structures with a different number of nanorods per primitive unit cell and volume fraction occupied by the nanorods by varying the angle of incidence and polarization direction of the incoming wave.

\section{METHOD OF CALCULATION}

Our calculations are based on the full-electrodynamic LMS method, ${ }^{43-45}$ which is ideally suited for stratified structures consisting of successive layers of nonoverlapping particles (scatterers) of arbitrary shape, arranged with the same 2D periodicity in the $x-y$ plane, whereas periodicity in the $z$ direction is not a prerequisite. The method solves Maxwell equations in the frequency domain, and thus dispersion and absorptive losses in the constituent materials can be readily included. Besides the complex photonic band structure of an infinite crystal, associated with a given crystallographic plane, the LMS method can also provide the reflectance, transmittance, and absorbance of a finite slab of the crystal at any angle of incidence, and, in this respect, it can describe an actual transmission experiment.

In the spirit of the multiple-scattering approach, the scattering properties of the entire composite structure are obtained from those of its constituent building units. At a first step, in-plane multiple scattering is described in a sphericalwave basis using the scattering $T$ matrix of the individual scatterers and appropriate propagator functions. For particles of cylindrical symmetry and arbitrary orientation, such as the nanorods that we consider in the present work, the $T$ matrix is first calculated by the extended-boundary-condition method in a coordinate system defined by taking the $z$ axis along the particle axis; then, it is transformed into the actual coordinate system using the relevant transformation matrices for the appropriate Euler rotation angles. ${ }^{46}$ Very good convergence of the calculations in all cases we studied here is obtained if we truncate the spherical-wave expansion at $l_{\max }=8$ and evaluate the single-particle scattering $T$ matrix with $l_{\text {cut }}=15$ and a Gaussian quadrature integration formula with 1024 points. 45,46 In a second step, interlayer coupling is fully taken into account by properly combining the transmission and reflection matrices of the component layers, so as to describe multiple scattering between the layers to any order in a plane-wave representation defined as follows. The component of the wavevector of the incident plane wave parallel to the layers, $\mathbf{q}_{\|}$, is written as $\mathbf{q}_{\|}=$ $\mathbf{k}_{\|}+\mathbf{g}^{\prime}$, where $\mathbf{k}_{\|}$, the reduced wavevector in the surface Brillouin zone, is a conserved quantity in the scattering process and $\mathbf{g}^{\prime}$ is a certain reciprocal vector of the given $2 \mathrm{D}$ lattice. Therefore, the wavevector of the incident wave has the form $\mathbf{K}_{\mathrm{g}^{\prime}}^{ \pm}$ $=\mathbf{k}_{\|}+\mathbf{g}^{\prime} \pm\left[q^{2}-\left(\mathbf{k}_{\|}+\mathbf{g}^{\prime}\right)^{2}\right]^{1 / 2} \hat{\mathbf{e}}_{z}$, where $q$ is the wavenumber, $\hat{\mathbf{e}}_{z}$ is the unit vector along the $z$ axis, and the + or - sign refers to incidence from $z<0$ or from $z>0$, that is, a wave propagating toward the positive or negative $z$ direction, respectively. Because $\mathbf{k}_{\|}$and the angular frequency $\omega$ are conserved quantities in the elastic scattering process, the scattered field consists of a series of plane waves with wavevectors $\mathbf{K}_{\mathrm{g}}^{ \pm}=\mathbf{k}_{\|}+\mathbf{g} \pm\left[q^{2}-\left(\mathbf{k}_{\|}+\mathbf{g}\right)^{2}\right]^{1 / 2} \hat{\mathbf{e}}_{z}, \forall \mathbf{g}$, and polarizations along $\hat{\mathbf{e}}_{1}$ and $\hat{\mathbf{e}}_{2}$ (polar and azimuthal unit vectors, respectively, associated with every $\mathbf{K}_{\mathrm{g}}^{ \pm}$). Corresponding circularly polarized plane waves are defined by the unit vectors $\hat{\mathbf{e}}_{\mathrm{L}}=\left(\hat{\mathbf{e}}_{1}+i \hat{\mathbf{e}}_{2}\right) / 2^{1 / 2}$ and $\hat{\mathbf{e}}_{\mathrm{R}}=\left(\hat{\mathbf{e}}_{1}-i \hat{\mathbf{e}}_{2}\right) / 2^{1 / 2}$ for left circular polarization (LCP) and right circular polarization (RCP), respectively. It is worth noting that although the scattered field consists, in general, of a number of diffracted beams corresponding to different $2 \mathrm{D}$ reciprocal lattice vectors $\mathrm{g}$, only beams for which $K_{\mathrm{gz}}^{ \pm}$is real constitute propagating waves. When $\left(\mathbf{k}_{\|}+\mathbf{g}\right)^{2}>q^{2}$, we have an evanescent beam, and the corresponding unit vectors $\hat{\mathbf{e}}_{1}, \hat{\mathbf{e}}_{2}$ become complex, but they are still orthonormal: $\hat{\mathbf{e}}_{p} \cdot \hat{\mathbf{e}}_{p^{\prime}}=\delta_{p p^{\prime}} p\left(p^{\prime}\right)=1,2$. Taking into account in the calculations $892 \mathrm{D}$ reciprocal lattice vectors ensures good convergence in all of the cases we studied in the present work.

The ratio of the transmitted or reflected energy flux to the energy flux associated with the incident wave defines the transmittance or reflectance, respectively, of a multilayer slab. For a 3D crystal consisting of an infinite periodic sequence of layers, stacked along the $z$ direction, applying the Bloch condition for the wave field in the region between two consecutive unit slabs leads to an eigenvalue equation, which gives the $z$ component of the Bloch wave vector, $k_{z}$, for the given $\omega$ and $\mathbf{k}_{\|}$. The eigenvalues $k_{z}\left(\omega, \mathbf{k}_{\|}\right)$, looked upon as functions of real $\omega$, define, for each $\mathbf{k}_{\|}$, lines in the complex $k_{z}$ plane. Taken together, they constitute the complex band structure of the infinite crystal associated with the given crystallographic plane. A line of given $\mathbf{k}_{\|}$may be real (in the sense that $k_{z}$ is real) over certain frequency regions and be complex (in the sense that $k_{z}$ is complex) for $\omega$ outside these regions. It turns out that for given $\mathbf{k}_{\|}$and $\omega$, out of the eigenvalues $k_{z}\left(\omega, \mathbf{k}_{\|}\right)$, none or, at best, a few are real, and the corresponding eigenvectors represent propagating modes of the electromagnetic (EM) field in the given infinite crystal. The remaining eigenvalues $k_{z}\left(\omega, \mathbf{k}_{\|}\right)$are complex, and the corresponding eigenvectors represent evanescent waves. These have an amplitude that increases exponentially in the positive or negative $z$ direction and, unlike the propagating waves, do not exist as physical entities in the infinite crystal. However, they are an essential part of the physical solutions of the EM field in a slab of finite thickness. A region of frequency where propagating waves do not exist, for given $\mathbf{k}_{\|}$, constitutes a frequency gap of the EM field for the given $\mathbf{k}_{\|}$. If over a frequency region no propagating wave exists whatever the value of $\mathbf{k}_{\|}$, then this region constitutes an absolute frequency gap.

\section{FORMATION OF HYBRID PLASMON MODES}

We consider layer-by-layer structures of metallic nanorods of length $L$, with a circular cross section of diameter $D$, stacked along the $z$ direction. In each layer, the nanorods are centered at the sites of a square lattice, of lattice constant $a$, with their axes aligned in a direction perpendicular to the $z$ axis. The nanorods in consecutive layers are mutually twisted through an angle $\varphi(\varphi=\pi / 2, \pi / 3, \pi / 4)$ and are separated by a distance $h$, equal to one tenth of their diameter, as shown in Figure 1. Therefore, the period $d$ of the structure along the $z$ direction comprises $N=\pi / \varphi$ layers and $d=1.1 N D$. To begin with, we shall describe the metallic material by the simple yet effective Drude dielectric function ${ }^{48}$ 


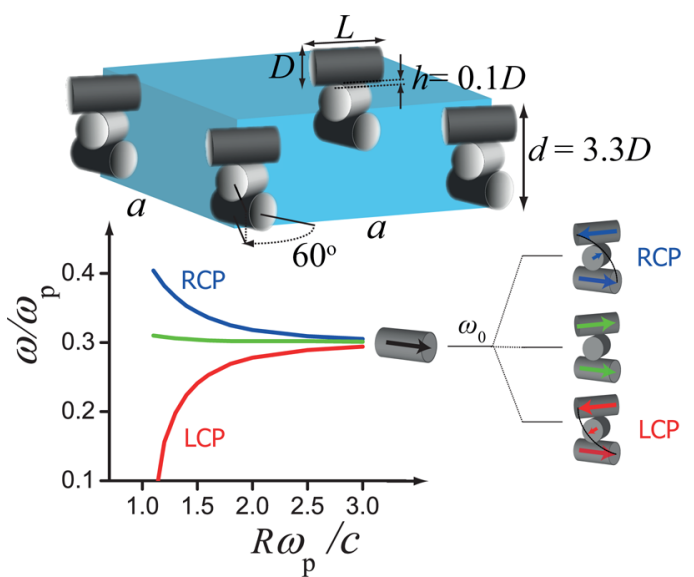

Figure 1. Unit cell of a tetragonal structure with a basis of three mutually twisted metallic nanorods and a schematic view of the formation of hybrid plasmon modes in the three-nanorod helical metamolecule. The bottom left diagram shows the variation of the eigenfrequencies of these modes as a function of the interparticle distance, as obtained by the point-dipole model described in the text. The calculated imaginary part of the eigenfrequencies is on the order of $10^{-3} \omega_{p}$.

$$
\varepsilon_{m}(\omega)=1-\frac{\omega_{p}^{2}}{\omega\left(\omega+i \tau^{-1}\right)}
$$

where $\omega_{p}$ is the bulk plasma frequency and $\tau$ is the relaxation time of the conduction band electrons, and take $D=c / \omega_{p}, L=$ $2.5 c / \omega_{p}$, and $a=7.5 c / \omega_{p}$. We note that assuming $\hbar \omega_{p} \cong 10 \mathrm{eV}$, the diameter of the nanorods, $D$, corresponds to $\sim 20 \mathrm{~nm}$ and their length, $L$, corresponds to $\sim 50 \mathrm{~nm}$.

The nature of the eigenmodes of the EM field in these structures can be better understood by reference to appropriate hybrid plasmon modes of the structural basis of twisted nanorods. The formation of these modes can be explained by a simple model of interacting point dipoles, which enables physical insight, as follows. The optical response of a metallic nanorod is characterized by a predominant resonance, which stems from the excitation of the fundamental dipole-like plasmon mode associated with free-electron oscillations along the nanorod axis (longitudinal mode). About the eigenfrequency of its longitudinal dipole particle-plasmon mode, a metallic nanorod can be assimilated to a point dipole oscillating in the direction of the rod axis. The electric field at $\mathbf{r}=r \hat{\mathbf{r}}$ from $\mathrm{a}$ point dipole $\mathrm{p}$ that oscillates with an angular frequency, $\omega$, assuming an $\exp (-i \omega t)$ time dependence is ${ }^{49}$

$$
\begin{aligned}
\mathbf{E}(\mathbf{r})= & {\left[\left(1-\frac{i \omega r}{c}\right) \frac{(\hat{\mathbf{r}} \cdot \mathbf{p}) \hat{\mathbf{r}}-\mathbf{p}}{r^{3}}+\frac{\omega^{2}}{c^{2}} \frac{\mathbf{p}-(\hat{\mathbf{r}} \cdot \mathbf{p}) \hat{\mathbf{r}}}{r}\right] } \\
& \exp (i \omega r / c)
\end{aligned}
$$

Therefore, for a collection of $N$ dipoles $\mathbf{p}_{j}$ at sites $\mathbf{R}_{j}=\left(0,0, z_{j}\right), j$ $=1, \ldots, N$, oscillating normal to the $z$ axis, the electric field on the $i$ th dipole, created by all other dipoles, is given by

$$
\mathbf{E}_{i}=\sum_{j, j \neq i} f\left(\omega,\left|\mathbf{R}_{i}-\mathbf{R}_{j}\right|\right) \mathbf{p}_{j}
$$

where

$$
f(\omega, R)=-\frac{1}{R^{3}}\left[1-\frac{i \omega R}{c}-\frac{\omega^{2} R^{2}}{c^{2}}\right] \exp (i \omega R / c)
$$

The dipole moment in the direction of the rod axis is given by the product of the longitudinal element of the particle electric polarizability tensor, $\alpha_{\|}$, and the component of the electric field along this direction

$$
p_{i}=\alpha_{\|} \mathbf{E}_{i} \cdot \hat{\mathbf{p}}_{i}
$$

Unfortunately, no closed-form expressions exist for the polarizability of a rod. However, it has been shown by means of numerical calculations that the polarizability of a rod is close to that of a spheroid with the same aspect ratio and permittivity, that is 50

$$
\alpha_{\|}(\omega)=\frac{V}{4 \pi} \frac{\varepsilon_{m}(\omega)-1}{1+L_{\|}\left[\varepsilon_{m}(\omega)-1\right]}
$$

where $V$ is the volume of the particle and $L_{\|}\left(0<L_{\|}<1\right)$ is the longitudinal depolarization factor. The eigenfrequency, $\omega_{0}$, of the longitudinal dipole particle-plasmon mode of a single nanorod is determined from the condition $\alpha_{\|}^{-1}\left(\omega_{0}\right)=0$. For example, if we assume that $\varepsilon_{m}(\omega)$ has the simple Drude form of eq 1 without dissipative losses $\left(\tau^{-1}=0\right)$, then we obtain $\omega_{0}=$ $\left(L_{\|}\right)^{1 / 2} \omega_{p}$. The scattering and absorption cross sections of a single nanorod made of a Drude metal, with $D=c / \omega_{p}$ and $L=$ $2.5 c / \omega_{p}$, and also of a corresponding nanorod made of actual silver $(D=20 \mathrm{~nm}$ and $L=50 \mathrm{~nm})$ have been presented and analyzed in detail elsewhere. ${ }^{51}$ They are characterized by a strong resonance at $\omega_{0}=0.3 \omega_{p}$ or $\hbar \omega_{p}=2.5 \mathrm{eV}$, respectively, that stems from the excitation of the fundamental longitudinal plasmon mode, from which we deduce $L_{\|}=0.09$.

For three interacting nanorods, separated by a distance $R=D$ $+h\left(=1.1 c / \omega_{p}\right)$ and mutually twisted through an angle $\varphi=\pi / 3$ as shown in Figure 1, eqs 3 and 5 lead to a linear system of equations, which can be cast into the form of an eigenvalue problem

$\left(\begin{array}{ccc}0 & f_{1}(\omega) & f_{2}(\omega) \\ f_{1}(\omega) & 0 & f_{1}(\omega) \\ f_{2}(\omega) & f_{1}(\omega) & 0\end{array}\right)\left(\begin{array}{c}p_{1} \\ p_{2} \\ p_{3}\end{array}\right)=\left[L_{\|}-\left(\frac{\omega}{\omega_{p}}\right)^{2}\right]\left(\begin{array}{c}p_{1} \\ p_{2} \\ p_{3}\end{array}\right)$

where $f_{1}(\omega)=(V / 4 \pi) f(\omega, R) \cos \varphi$ and $f_{2}(\omega)=(V / 4 \pi) f(\omega, 2 R)$ $\cos 2 \varphi$. The eigenvalues and eigenvectors of the matrix on the left-hand side of eq 7 are analytically given by: $\left[f_{2}(\omega)-\right.$ $\left.\left(8 f_{1}^{2}(\omega)+f_{2}^{2}(\omega)\right)^{1 / 2}\right] / 2,-f_{2}(\omega),\left[f_{2}(\omega)+\left(8 f_{1}^{2}(\omega)+\right.\right.$ $\left.\left.f_{2}^{2}(\omega)\right)^{1 / 2}\right] / 2$, and $\left(1,\left[-f_{2}(\omega)-\left(8 f_{1}^{2}(\omega)+f_{2}^{2}(\omega)\right)^{1 / 2}\right] /\right.$ $\left.2 f_{1}(\omega), 1\right)^{T},(1,0,-1)^{T}$, and $\left(1,\left[-f_{2}(\omega)+\left(8 f_{1}^{2}(\omega)+\right.\right.\right.$ $\left.\left.\left.f_{2}^{2}(\omega)\right)^{1 / 2}\right] / 2 f_{1}(\omega), 1\right)^{T}$, respectively. Setting these eigenvalues equal to $0.09-\left(\omega / \omega_{p}\right)^{2}$, we obtain the eigenfrequencies and the associated hybrid plasmon modes of the three-nanorod helical metamolecule, shown in Figure 1. It can be seen that interaction between the nanorods leads to the formation of three distinct hybrid modes: one of LCP-like character, one of RCP-like character, and one with no specific circular-polarization character. It is also worth noting that these modes have a finite lifetime because of radiative losses.

Similarly, for four interacting nanorods, mutually twisted through an angle $\varphi=\pi / 4$, we obtain four hybrid modes: one of LCP-like character, one of RCP-like character, and two with no specific circular-polarization character, whereas for a pair of nanorods, mutually twisted through an angle $\varphi=\pi / 2$, neither of the two hybrid modes formed has a specific circularpolarization character. 


\section{PHOTONIC BAND STRUCTURE AND TRANSMISSION SPECTRA}

In Figure 2, we display photonic dispersion diagrams of the three crystals under consideration for $\mathbf{k}_{\|}=(0.25,0) 2 \pi / a$,
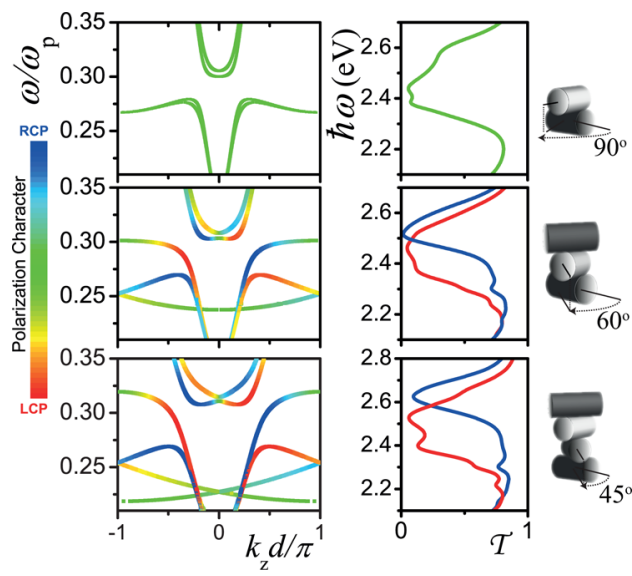

Figure 2. Photonic band structure of the crystals under study with a basis of two (top diagram), three (middle diagram), and four (bottom diagram) metallic nanorods per unit cell, arranged as shown in Figure 1 for a specific case, for $\mathbf{k}_{\|}=(0.25,0) 2 \pi / a$. The dielectric function of the metal is described by the Drude model without dissipative losses (eq 1 with $\tau^{-1}=0$ ), and the geometric parameters of the structures are: $D=c / \omega_{p}, L=2.5 c / \omega_{p}$, and $a=7.5 c / \omega_{p}$. Next to the band diagrams, we depict corresponding transmission spectra of (001) slabs of these crystals, 12-layers thick, for LCP and RCP light incident along the positive $z$ direction if we consider silver nanorods described by the experimental dielectric function of bulk silver ${ }^{52}$ and geometric parameters of the structures: $D=20 \mathrm{~nm}, L=50 \mathrm{~nm}$, and $a=150 \mathrm{~nm}$.

calculated by deliberately disregarding absorptive losses taking $\tau^{-1}=0$ in eq 1 to ensure an unambiguous interpretation. Along an arbitrary direction of the Brillouin zone, such as the one we consider here, all bands are nondegenerate because they belong to the identity representation of the trivial group and thus they cannot be classified as of purely LCP or RCP character. However, there is a different degree of LCP and RCP admixture that varies along a specific band (see middle and bottom band diagrams of Figure 2), except for the case of $\varphi=$ $\pi / 2$ (top band diagram of Figure 2), where chirality is absent and the bands have no predominant circular-polarization character. The circular-polarization character of a given Bloch mode, presented by the color scale in Figure 2, is defined by projecting the corresponding eigenvector ${ }^{43-46}$ onto the basis spanned by the LCP and RCP states, associated with $\hat{\mathbf{e}}_{\mathrm{L}}$ and $\hat{\mathbf{e}}_{\mathrm{R}}$ respectively. The dispersion diagrams of Figure 2 are characterized by the presence of two extended bands, associated with photons of two transverse orthogonal polarizations propagating in an underlying effective medium, and a number of relatively narrow bands, as many as the number of nanorods per primitive cell, formed by the interacting molecular plasmonic orbitals, which stem from the fundamental longitudinal dipole-like plasmon modes of the individual nanorods, as described in the previous section. Symmetry compatibility allows for anticrossing interaction between the bands, which always takes place to a major or minor degree depending on the shape of the modes involved and leads to the appearance of frequency gaps. In a chiral structure, the two extended effective-medium bands and two of the narrow plasmonic bands have a predominant circular-polarization character, one of each being LCP-like and one RCP-like. Anticrossing interaction between extended and narrow bands of the same character leads to strong level repulsion and consequent sizable frequency gaps for a specific polarization, as can be seen in the middle and bottom band diagrams of Figure 2.

Characterizing the band eigenmodes by their degree of LCP and RCP admixture allows for a consistent interpretation of corresponding transmission spectra of finite slabs, as shown in the right-hand diagrams of Figure 2. Modes with a certain predominant circular-polarization character and positive (negative) group velocity couple predominantly to a plane EM wave with the same polarization, incident along the positive (negative) $z$ direction on a (001) slab of the crystal, with the same $\mathbf{k}_{\|}$. As shown in the middle and bottom transmission diagrams of Figure 2, over the frequency range of a polarization gap, only incident waves of opposite handedness are allowed to pass through. Of course, for the crystal with a basis of two nanorods where chirality is absent, the transmission spectra for LCP and RCP incident light are the same. In a terminology similar to that introduced by Hodgkinson et al. ${ }^{53}$ in relation to chiral layered structures of homogeneous anisotropic plates, this crystal can be classified as an equichiral layered structure, whereas the crystals with a basis of three and four nanorods can be classified as ambichiral layered structures. It is worth noting that the position of the circular-polarization spectral gaps, which are relevant here, is determined by the localized particleplasmon modes and thus can be easily tuned by changing the rod length, ${ }^{51}$ contrary to the circular Bragg gaps in, for example, cholesteric liquid crystals and chiral sculptured thin films, which appear at wavelengths commensurate with the lattice constant.

\section{OPTICAL ACTIVITY}

The polarization state of a wave transmitted through a finite slab of the crystals under consideration can be directly obtained from the corresponding transmission matrix. It is worth noting that the frequency region of interest is below the diffraction limit for light incident on the (001) surface of these crystals, at any angle, as appropriate for the metamaterial regime. Therefore, only the zero-order diffraction channel yields a propagating beam, and the (electric-field) amplitude of the transmitted wave is obtained from that of the incident wave through a $2 \times 2$ complex transmission matrix, $\mathbf{t}$, as follows: $E_{p}^{\mathrm{tr}}=$ $\Sigma_{p^{\prime}} t_{p p^{\prime}} E_{p^{\prime}}^{\text {in }}$, where $p\left(p^{\prime}\right)=1,2$ refers to linearly polarized waves with the electric field oscillating in or perpendicular to the plane of incidence, respectively, in accordance with the plane-wave basis, spanned by the unit vectors $\hat{\mathbf{e}}_{1}$ and $\hat{\mathbf{e}}_{2}$, employed in the LMS method. We note that at normal incidence on the (001) surface of the crystal, these polarization directions coincide with the $x$ and $y$ axes, respectively. One can easily switch from this basis of linearly polarized waves $\left[p\left(p^{\prime}\right)=1,2\right]$ to the basis of circularly polarized waves $\left[p\left(p^{\prime}\right)=\mathrm{L}, \mathrm{R}\right]$ with the help of a similarity transformation

$$
\left(\begin{array}{ll}
t_{\mathrm{LL}} & t_{\mathrm{LR}} \\
t_{\mathrm{RL}} & t_{\mathrm{RR}}
\end{array}\right)=\frac{1}{\sqrt{2}}\left(\begin{array}{cc}
1 & -i \\
1 & i
\end{array}\right)\left(\begin{array}{ll}
t_{11} & t_{12} \\
t_{21} & t_{22}
\end{array}\right)\left[\frac{1}{\sqrt{2}}\left(\begin{array}{cc}
1 & -i \\
1 & i
\end{array}\right)\right]^{-1}
$$

An incoming plane wave of amplitude $E_{0}$, propagating along the positive $z$ direction, linearly polarized at an angle $\phi_{0}$ with respect to the $\hat{\mathbf{e}}_{1}$ axis, can be decomposed into LCP and RCP waves of amplitudes $E_{\mathrm{L}}^{\text {in }}=E_{0} \exp \left(-i \phi_{0}\right) / 2^{1 / 2}$ and $E_{\mathrm{R}}^{\text {in }}=E_{0}$ $\exp \left(i \phi_{0}\right) / 2^{1 / 2}$, respectively. The corresponding transmitted fields have amplitudes $E_{\mathrm{L}}^{\mathrm{tr}}=E_{0} / 2^{1 / 2}\left[t_{\mathrm{LL}} \exp \left(-i \phi_{0}\right)+t_{\mathrm{LR}}\right.$ 
$\left.\exp \left(i \phi_{0}\right)\right]$ and $E_{\mathrm{R}}^{\mathrm{tr}}=E_{0} / 2^{1 / 2}\left[t_{\mathrm{RL}} \exp \left(-i \phi_{0}\right)+t_{\mathrm{RR}} \exp \left(i \phi_{0}\right)\right]$, which correspond to an in general elliptically polarized wave with the long axis of the ellipse forming an angle

$$
\phi=\frac{1}{2}\left[\arg \left(E_{\mathrm{R}}^{\mathrm{tr}}\right)-\arg \left(E_{\mathrm{L}}^{\mathrm{tr}}\right)\right]
$$

with the $\hat{\mathbf{e}}_{1}$ axis and with ellipticity angle

$$
\chi=\arctan \frac{\left|E_{\mathrm{R}}^{\mathrm{tr}}\right|-\left|E_{\mathrm{L}}^{\mathrm{tr}}\right|}{\left|E_{\mathrm{R}}^{\mathrm{tr}}\right|+\left|E_{\mathrm{L}}^{\mathrm{tr}}\right|}
$$

as shown in Figure 3. We adopt the polarization azimuth rotation angle, $\Delta \phi=\phi-\phi_{0}$, as a measure of circular birefringence and the ellipticity angle, $\chi$, as a measure of circular dichroism.

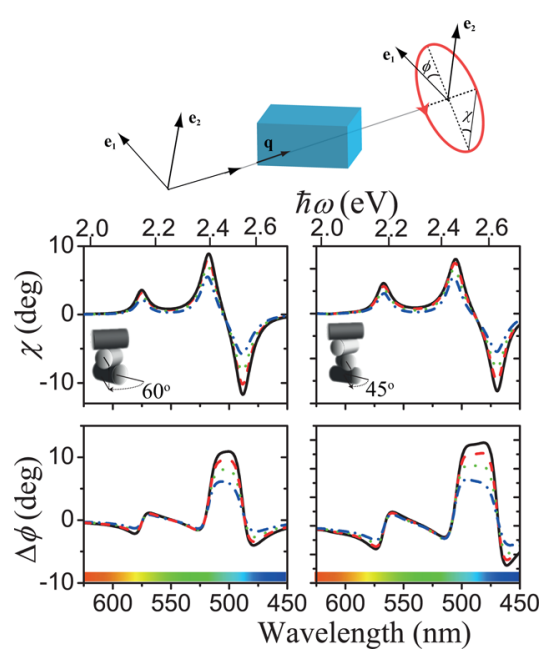

Figure 3. Variation of the polarization-averaged ellipticity (upper diagrams) and azimuth rotation (lower diagrams) angles of a wave transmitted through a (001) unit slab of the crystal with a basis of three silver nanorods (left-hand diagrams) and of the crystal with a basis of four nanorods (right-hand diagrams) for different angles of incidence: $0^{\circ}$ (solid line), $30^{\circ}$ (dashed line), $45^{\circ}$ (dotted line), and $60^{\circ}$ (dashed-dotted line) in the visible spectrum. The nanorods are described by the experimental dielectric function of bulk silver, ${ }^{52}$ and the geometric parameters of the structures are: $D=20 \mathrm{~nm}, L=50 \mathrm{~nm}$, and $a=150 \mathrm{~nm}$.

It is worth noting that, contrary to the situation under, for example, a four-fold rotation symmetry, in our case there is polarization conversion because $t_{\mathrm{LR}}$ and $t_{\mathrm{RL}}$ do not vanish identically. As a result, $\Delta \phi$ and $\chi$ vary with the polarization angle $\phi_{0}$ in an oscillatory manner, with a period of $180^{\circ}$. In Figure 3 , we display the calculated polarization-averaged ellipticity and azimuth rotation angles of a wave transmitted through a (001) unit slab of the crystals with a basis of three and four nanorods (three- and four-layers thick, respectively) for different angles of incidence. In the former (latter) case, the polarization-averaged ellipticity angle vanishes at $\hbar \omega=$ 2.46(2.54) eV, for all different angles of incidence, which indicates a pure optical activity effect. At this frequency, the polarization-averaged azimuth rotation of the transmitted wave decreases from $10.8^{\circ}\left(14.4^{\circ}\right)$ at normal incidence to $6^{\circ}\left(8^{\circ}\right)$ for incidence at an angle of $60^{\circ}$. In terms of rotatory power per sample thickness equal to one wavelength, the optical activity of the considered unit slabs can be as large as $83^{\circ}\left(80^{\circ}\right)$, which is by several orders of magnitude larger than that of naturally occurring optically active materials and comparable to that of other chiral metamaterials, ${ }^{22,54}$ whereas transmission losses are relatively low, typically a few decibels. Stronger optical activity effects are obtained for more dense structures. For example, the rotatory power becomes twice larger if the lattice constant $a$ is reduced from 150 to $100 \mathrm{~nm}$. It is also worth noting that the spectral response can be tuned in a controllable manner by choosing the rod length so as to shift its plasmon resonance appropriately. ${ }^{51}$

\section{CONCLUSIONS}

In conclusion, we reported a systematic study of the optical properties of a series of layer-by-layer chiral periodic structures consisting of twisted silver nanorods. We analyzed photonic band diagrams of these crystals in conjunction with relevant polarization-resolved transmission spectra of corresponding finite slabs and explained the formation of partial gaps for a specific circular polarization, by reference to the hybrid plasmon modes of the structural basis of twisted nanorods. We demonstrated the occurrence of giant optical activity effects, which can be tuned within the infrared and visible spectrum by appropriately choosing the rod length and persist for any angle of incidence and polarization direction. These effects do not strongly depend on the twisting angle of the nanorods and become more pronounced with increasing particle concentration. All of these features, along with relatively low transmission losses, make few-layer slabs of these crystals promising candidates for practical applications as ultrathin circular polarizers and polarization rotators. Because of the localized nature of the plasmon modes and the strong chiral response of the structures under consideration along any direction, enhanced optical activity effects should also be expected for helical complexes of metallic nanorods randomly distributed in solution.

\section{AUTHOR INFORMATION}

\section{Corresponding Author}

*E-mail: aristi@ims.demokritos.gr.

\section{Notes}

The authors declare no competing financial interest.

\section{ACKNOWLEDGMENTS}

A.C. is supported by NCSR "Demokritos" through a postgraduate fellowship.

\section{REFERENCES}

(1) Berova, N.; Nakanishi, K.; Woody, R. W. Circular Dichroism: Principles and Applications, 2nd ed.; Wiley-VCH: New York, 2000.

(2) de Gennes, P. G.; Prost, J. The Physics of Liquid Crystals, 2nd ed.; Clarendon Press: Oxford, U.K., 1993.

(3) Kopp, V. I.; Fan, B.; Vithana, H. K. M.; Genack, A. Z. Opt. Lett. 1998, 23, 1707-1709.

(4) Hwang, J.; Song, M. H.; Park, B.; Nishimura, S.; Toyooka, T.; Wu, J. W.; Takanishi, Y.; Ishikawa, K.; Takezoe, H. Nat. Mater. 2005, 4, 383-387.

(5) Karathanos, V.; Stefanou, N.; Modinos, A. J. Mod. Opt. 1995, 42, 619-626.

(6) Robbie, K.; Brett, M. J.; Lakhtakia, A. Nature (London) 1996, 384, 616.

(7) Hodgkinson, I.; Wu, Q. H.; Knight, B.; Lakhtakia, A.; Robbie, K. Appl. Opt. 2000, 39, 642-649.

(8) Pang, Y. K.; Lee, J.; Lee, H.; Tam, W. Y.; Chan, C.; Sheng, P. Opt. Express 2005, 13, 7615-7620.

(9) Lee, J.; Chan, C. Opt. Express 2005, 13, 8083-8088. 
(10) Hrudey, P. C. P.; Szeto, B.; Brett, M. J. Appl. Phys. Lett. 2006, 88,251106

(11) Furumi, S.; Sakka, Y. Adv. Mater. 2006, 18, 775-780.

(12) Mitov, M.; Dessaud, N. Nat. Mater. 2006, 5, 361-364.

(13) Thiel, M.; Decker, M.; Deubel, M.; Wegener, M.; Linden, S.; von Freymann, G. Adv. Mater. 2007, 19, 207-210.

(14) Gansel, J. K.; Thiel, M.; Rill, M. S.; Decker, M.; Bade, K.; Saile, V.; von Freymann, G.; Linden, S.; Wegener, M. Science 2009, 325, 1513-5.

(15) Papakostas, A.; Potts, A.; Bagnall, D. M.; Prosvirnin, S. L.; Coles,

H. J.; Zheludev, N. I. Phys. Rev. Lett. 2003, 90, 107404-1.

(16) Vallius, T.; Jefimovs, K.; Turunen, J.; Vahimaa, P.; Svirko, Y. Appl. Phys. Lett. 2003, 83, 234-236.

(17) Kuwata-Gonokami, M.; Saito, N.; Ino, Y.; Kauranen, M.; Jefimovs, K.; Vallius, T.; Turunen, J.; Svirko, Y. Phys. Rev. Lett. 2005, 95, 227401.

(18) Plum, E.; Fedotov, V. A.; Schwanecke, A. S.; Zheludev, N. I.; Chen, Y. Appl. Phys. Lett. 2007, 90, 223113.

(19) Decker, M.; Klein, M. W.; Wegener, M.; Linden, S. Opt. Lett. 2007, 32, 856-858.

(20) Konishi, K.; Sugimoto, T.; Bai, B.; Svirko, Y.; KuwataGonokami, M. Opt. Express 2007, 15, 9575-9583.

(21) Kwon, D. -H.; Werner, P. L.; Werner, D. H. Opt. Express 2008, $16,11802-11807$.

(22) Helgert, C.; Pshenay-Severin, E.; Falkner, M.; Menzel, C.; Rockstuhl, C.; Kley, E. -B.; Tünnermann, A.; Lederer, F.; Pertsch, T. Nano Lett. 2011, 11, 4400-4404.

(23) Fan, Z.; Govorov, A. O. Nano Lett. 2010, 10, 2580-2587.

(24) Fan, Z.; Govorov, A. O. J. Phys. Chem. C 2011, 115, 1325413261.

(25) Auguié, B.; Alonso-Gómez, J. L.; Guerrero-Martínez, A.; LizMarzán, L. M. Phys. Chem. Lett. 2011, 2, 846-851.

(26) Guerrero-Martínez, A.; Auguié, B.; Alonso-Gómez, J. L.; Džolić, Z.; Gómez-Graña, S.; Žinić, M.; Cid, M. M.; Liz-Marzán, L. M. Angew. Chem., Int. Ed. 2011, 123, 5613-5617.

(27) Guerrero-Martínez, A.; Alonso-Gómez, J. L.; Auguié, B.; Cid, M. M.; Liz-Marzán, L. M. Nano Today 2011, 6, 381-400.

(28) Gao, W.; Leung, H. M.; Li, Y.; Chen, H.; Tam, W. Y. J. Opt. 2011, 13, 115101.

(29) Hentschel, M.; Schäferling, M.; Weiss, T.; Liu, N.; Giessen, H. Nano Lett. 2012, 12, 2542-2547.

(30) Zhao, Y.; Belkin, M. A.; Alú, A. Nat. Commun. 2012, 3, 870.

(31) Chirality at the Nanoscale; Amabilino, D. B., Ed.; Wiley-VCH: New York, 2008.

(32) Berova, N.; Bari, L. D.; Pescitelli, G. Chem. Soc. Rev. 2007, 36, 914-931.

(33) Chen, W.; Bian, A.; Agarwal, A.; Liu, L.; Shen, H.; Wang, L.; Xu, C.; Kotov, N. A. Nano Lett. 2009, 9, 2153-2159.

(34) Soukoulis, C. M.; Wegener, M. Nat. Photon. 2011, 5, 523-530. (35) Fan, J. A.; Wu, C.; Bao, K.; Bao, J.; Bardhan, R.; Halas, N. J.; Manoharan, V. N.; Nordlander, P.; Shvets, G.; Capasso, F. Science 2010, 328, 1135-1138.

(36) Jones, M. R.; Osberg, K. D.; Macfarlane, R. J.; Langille, M. R.; Mirkin, C. A. Chem. Rev. 2011, 111, 3736-827.

(37) Mastroianni, A. J.; Claridge, S. A.; Alivisatos, A. P. J. Am. Chem. Soc. 2009, 131, 8455-8459.

(38) Sharma, J.; Chhabra, R.; Cheng, A.; Brownell, J.; Liu, Y.; Yan, H. Science 2009, 323, 112-6.

(39) Tan, S. J.; Campolongo, M. J.; Luo, D.; Cheng, W. Nat. Nanotechnol. 2011, 6, 268-276.

(40) Shen, X.; Song, C.; Wang, J.; Shi, D.; Wang, Z.; Liu, N.; Ding, B. J. Am. Chem. Soc. 2012, 134, 146-149.

(41) Kuzyk, A.; Schreiber, R.; Fan, Z.; Pardatscher, G.; Roller, E. -M.; Högele, A.; Simmel, F. C.; Govorov, A. O.; Liedl, T. Nature 2012, 483, $311-314$.

(42) Christofi, A.; Stefanou, N.; Gantzounis, G.; Papanikolaou, N. Phys. Rev. B 2011, 84, 125109.

(43) Stefanou, N.; Yannopapas, V.; Modinos, A. Comput. Phys. Commun. 1998, 113, 49-77.
(44) Stefanou, N.; Yannopapas, V.; Modinos, A. Comput. Phys. Commun. 2000, 132, 189-196.

(45) Gantzounis, G.; Stefanou, N. Phys. Rev. B 2006, 73, 035115.

(46) Mishchenko, M. I.; Travis, L. D.; Lacis, A. A. Scattering, Absorption, and Emission of Light by Small Particles; Cambridge University Press: Cambridge, U.K., 2002.

(47) Prodan, E.; Radloff, C.; Halas, N. J.; Nordlander, P. Science 2003, 302, 419-422.

(48) Ashcroft,N. W.; Mermin, N. D. Solid State Physics; Saunders: New York, 1976.

(49) Jackson, J. D. Classical Electrodynamics; Wiley: New York, 1999.

(50) Venermo, J.; Sihvola, A. J. Electrost. 2005, 63, 101-117.

(51) Tserkezis, C.; Papanikolaou, N.; Almpanis, E.; Stefanou, N. Phys. Rev. B 2009, 80, 125124.

(52) Johnson, P. B.; Christy, R. W. Phys. Rev. B 1972, 6, 4370-4379.

(53) Hodgkinson, I. J.; Lakhtakia, A.; Wu, Q. H.; De Silva, L.; McCall, M. W. Opt. Commun. 2004, 239, 353-358.

(54) Plum, E.; Zhou, J.; Dong, J.; Fedotov, V. A.; Koschny, T.; Soukoulis, C. M.; Zheludev, N. I. Phys. Rev. B 2009, 79, 035407. 